OPEN ACCESS

Edited by:

Brian Timothy Collins, Georgetown University School of

Medicine, United States

Reviewed by:

Vivek Verma,

University of Nebraska Medical

Center, United States

Michael Chuong,

Baptist Health South Florida

United States

*Correspondence:

Dwight E. Heron

herond2@upmc.edu

Specialty section: This article was submitted to

Radiation Oncology,

a section of the journal

Frontiers in Oncology

Received: 28 August 2017 Accepted: 30 October 2017

Published: 14 November 2017

Citation:

Sutera PA, Bernard ME, Gill BS, Harper KK, Quan K, Bahary N,

Burton SA, Zeh $H$ and Heron DE (2017) One- vs. Three-Fraction Pancreatic Stereotactic Body

Radiation Therapy for Pancreatic Carcinoma: Single Institution

Retrospective Review.

Front. Oncol. 7:272.

doi: 10.3389/fonc.2017.00272

\section{One- vs. Three-Fraction Pancreatic Stereotactic Body Radiation Therapy for Pancreatic Carcinoma: Single Institution Retrospective Review}

\author{
Philip Anthony Sutera', Mark E. Bernard ${ }^{2}$, Beant S. Gill', Kamran K. Harper'1, \\ Kimmen Quan', Nathan Bahary ${ }^{3}$, Steven A. Burton ${ }^{1}$, Herbert Zeh ${ }^{4}$ and Dwight E. Heron ${ }^{1 *}$ \\ ${ }^{1}$ Department of Radiation Oncology, Hillman Cancer Center, Pittsburgh, PA, United States, ${ }^{2}$ Department of Radiation \\ Medicine, University of Kentucky, Lexington KY, ${ }^{3}$ Department of Medical Oncology, Hillman Cancer Center, Pittsburgh, \\ PA, United States, ${ }^{4}$ Department of Surgical Oncology, Hillman Cancer Center, Pittsburgh, PA, United States
}

Background/introduction: Early reports of stereotactic body radiation therapy (SBRT) for pancreatic ductal adenocarcinoma (PDAC) used single fraction, but eventually shifted to multifraction regimens. We conducted a single institution review of our patients treated with single- or multifraction SBRT to determine whether any outcome differences existed.

Methods and materials: Patients treated with SBRT in any setting for PDAC at our facility were included, from 2004 to 2014. Overall survival (OS), local control (LC), regional control $(\mathrm{RC})$, distant metastasis $(\mathrm{DM})$, and late grade 3 or greater radiation toxicities from the time of SBRT were calculated using Kaplan-Meier estimation to either the date of last follow-up/death or local/regional/distant failure.

Results: We identified 289 patients (291 lesions) with pathologically confirmed PDAC. Median age was 69 (range, 33-90) years. Median gross tumor volume was 12.3 (8.621.3) $\mathrm{cm}^{3}$ and planning target volume $17.9(12-27) \mathrm{cm}^{3}$. Single fraction was used in 90 (30.9\%) and multifraction in 201 (69.1\%) lesions. At a median follow-up of 17.3 months (IQR 10.1-29.3 months), the median survival for the entire cohort 17.8 months with a 2 -year OS of $35.3 \%$. Univariate analysis showed multifraction schemes to have a higher 2-year OS $30.5 \%$ vs. $37.5 \%(p=0.019)$, it did not hold significance on MVA. Multifractionation schemes were found to have a higher LC on MVA $(\mathrm{HR}=0.53,95 \% \mathrm{Cl}$, 0.33-0.85, $p=0.009$ ). At 2 years, late grade $3+$ toxicity was $2.5 \%$. Post-SBRT CA19-9 was found on MVA to be a prognostic factor for OS $(\mathrm{HR}=1.01,95 \% \mathrm{Cl}, 1.01-1.01$, $p=0.009), \mathrm{RC}(\mathrm{HR}=1.01,95 \% \mathrm{Cl} 1.01-1.01, p=0.02)$, and $\mathrm{DM}(\mathrm{HR}=1.01,95 \% \mathrm{Cl}$, $1.01-1.01, p=0.001)$.

Conclusion: Our single institution retrospective review is the largest to date comparing single and multifraction SBRT and the first to show multifraction regimen SBRT to have a higher LC than single fractionation. Additionally, we show low rates of severe late toxicity with SBRT.

Keywords: pancreatic adenocarcinoma, stereotactic body radiation therapy, overall survival, local control, fractionation, toxicity 


\section{INTRODUCTION}

Pancreatic carcinoma is an aggressive malignancy with a predicted 53,070 new cases and 41,780 deaths in the US in 2016 with a 5 -year survival of only 7\% (1). Currently, tumor resectability is the single most powerful prognostic factor with surgery allowing the only practical chance for cure (2). Systemic chemotherapy and radiation, however, have been shown to play an important role as adjuvant therapy. Unfortunately, less than $20 \%$ of patients are deemed surgical candidates at time of presentation due to the commonly advanced presentation of disease $(3,4)$.

Historically, radiation therapy for pancreatic adenocarcinoma consisted of 6 weeks of external beam radiation therapy (EBRT) which was associated with significant toxicity and delayed time to systemically dosed chemotherapy (5). Drawing upon the success of stereotactic radiosurgery in the treatment of intracranial tumors, stereotactic body radiation therapy (SBRT) was developed in order to deliver a hypofractioned treatment to extracranial tumors $(6,7)$. Using high precision, this can deliver a high biological effective dose to the tumor while minimizing dose to surrounding tissue (8).

Recently, SBRT has demonstrated utility as primary treatment in unresectable disease, neoadjuvant treatment in locally advanced disease, and adjuvant therapy for resected and recurrent pancreatic tumors (9-14). Due to the shorter duration of treatment in SBRT over standard EBRT, patients receive full dose systemic chemotherapy with less delay. Importantly, studies show SBRT to have excellent local control (LC) and minimal toxicity rates while remaining a cost-effective treatment option (15-17). Initial experience with SBRT for pancreatic adenocarcinoma primarily used single fraction regimens however were complicated by relatively high rates of late GI toxicity $(11,18,19)$. Later experiences used multifraction regimens were implemented and have since been shown to limit late GI toxicity (20). Data comparing single- to multifraction regimens, however remain limited and are unclear whether there are any other differences in these treatments besides toxicity rates. We therefore aim to compare single to multifraction SBRT for pancreatic cancer to distinguish possible differences in control rates, overall survival (OS), and toxicity.

\section{MATERIALS AND METHODS}

\section{Patient Population}

Following approval from our institutional review board, we reviewed patients with histologically proven pancreatic adenocarcinoma treated with SBRT in either one or three fractions between 2004 and 2014. Patients received SBRT as neoadjuvant, adjuvant, or definitive treatment. Patients with resectable, borderline resectable, unresectable, medically inoperable, and recurrent tumors were included in this study. Patients with distant metastasis (DM) at diagnosis (who were provided SBRT for symptom control) were excluded as well as one additional patient excluded for having no records other than SBRT date and dose. Patients included in the study were staged clinically, with the use of CT scans and endoscopic ultrasound techniques. SBRT was performed on either a CyberKnife robotic radiosurgery (Accuray
Inc., Sunnyvale, CA, USA) or non-robotic linear accelerator based platforms (Trilogy, TrueBeam) (Varian Medical Systems, Palo Alto, CA, USA). Patient variables including age, race, gender, SMAD4 mutation, surgical status, chemotherapy treatment, prior EBRT, and SBRT dose, dosimetry, and advanced toxicities were collected.

\section{Definition of Parameters}

Resectable status was determined by a multidisciplinary case review using NCCN guidelines for resectable, borderline resectable, and unresectable disease. Local, regional, and distant progression were determined based on radiographic findings on follow up and/or confirmatory biopsy if done. Local progression was identified as progressive disease using RECIST 1.1 criteria. This is characterized by at least a $20 \%$ increase in the sum of diameters of the tumor with minimum of a $5 \mathrm{~mm}$ increase (21). Regional failure was defined as disease progression to the regional nodes defined as $\mathrm{n} 1, \mathrm{n} 2$, or $\mathrm{n} 3$ by the JPS classification $(22,23)$ (or new tumor growth within the pancreas outside of the radiation field). Toxicity was graded retroactively with the Common Terminology Criteria for Adverse Events Version 4.0 (CTCAE 4.0). Patients included in this review were simulated in the supine position using four-dimensional CT scan with IV contrast in a vacuum lock bag and wingboard. The $4 \mathrm{D}-\mathrm{CT}$ scan was obtained utilizing $1.25 \mathrm{~mm}$ slices simulated in a vacuum lock bag. During the time of simulation, a motion study was performed during which we obtained multiple images during the respiratory cycle using the abdominal marker as a surrogate for the respiratory cycle. The signal detected from the abdominal surrogate was used to bin the CT images, creating a series of separate CT scans for each phase in the breathing cycle. We then contoured the gross tumor volume (GTV) to see whether any motion was detected during the breathing cycles. If the motion was found to be more than $5 \mathrm{~mm}$, we decided to use respiratory gating. In this technique, we determined which phases of the breathing cycle limit the tumor motion to $5 \mathrm{~mm}$ and treat during those specific phases. During the patient's treatment, an equivalent abdominal surrogate signal is used to control the beam on time of the linear accelerator. The GTV was determined based on the simulation CT scan and diagnostic CT scans. The planning target volume (PTV) margin was added to be approximately $3 \mathrm{~mm}$ from GTV with editing off of the bowel (Figure 1). Patients included in the study had fiducials placed before CT-simulation to assist with target delineation during treatment. The bowel was our major dose limiting structured and was limited to no more than $30 \mathrm{~Gy}$ maximum. The max dose for the kidneys, liver, and cord were limited to 15,50 , and 15 Gy, respectively. Notably two patients exceeded the max dose for the right kidney (29.0 and 18.3 Gy), and one patient exceeded the max dose for the cord (27.6 Gy).

\section{Statistical Analysis}

Overall survival, LC, regional control (RC), DM, and advanced grade 3 or greater radiation toxicities from the time of SBRT were calculated using Kaplan-Meier estimation to either the date of last follow-up/death or local/regional/distant failure. Predictive factors for OS, LC, RC, DM, and advanced toxicities were determined through use of univariate log-rank test or Cox regression 


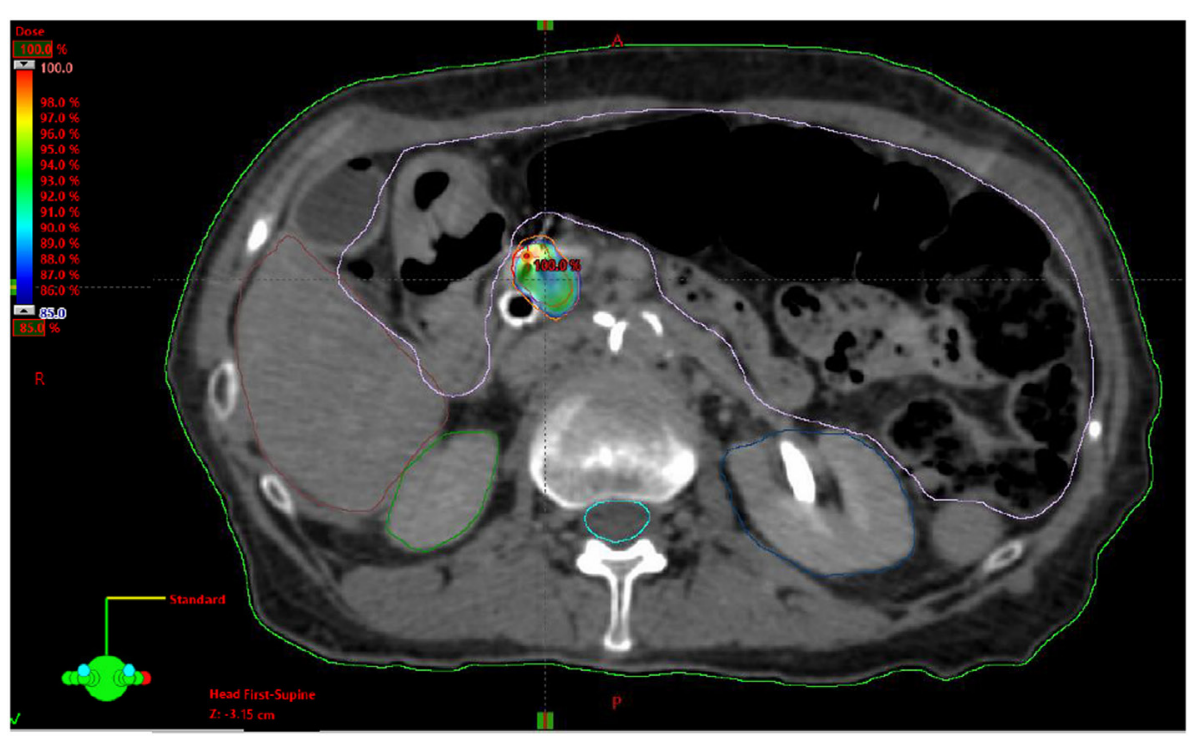

FIGURE 1 | Example stereotactic body radiation therapy plan for pancreatic adenocarcinoma with gross tumor volume highlighted in red, planning target volume highlighted in orange and $85 \%$ isodose highlighted in dose heatmap.

analysis. Those variables deemed significant $(p<0.05)$ were incorporated into multivariable survival analyses using forward stepwise selection in a Cox proportional hazards regression model. Statistical significance was set with a two-sided $p$-value of $<0.05$. Statistical analysis was performed using IBM SPSS Statistics version 23 .

\section{RESULTS}

\section{Patient Characteristics}

A detailed list of patient characteristics can be found in Table $\mathbf{1}$. We identified 289 patients with 291 lesions treated with SBRT of pancreatic adenocarcinoma with a median age at diagnosis of 69 (range 33-90) with $49.8 \%$ female and 50.2\% male. Tumors were located in the head $(62.9 \%)$, body $(11 \%)$, uncinate process (7.6\%), neck (4.8\%), tail (2.7\%), and genu $(0.3 \%)$ of the pancreas. Multifocal disease as defined by two or more lesions within the pancreas was seen in $10.7 \%$. SMAD4 mutation status was collected and positive in $10 \%$ and unknown in $78.7 \%$ of lesions. Recurrent lesions represented $13.4 \%$ of the total and $13.1 \%$ of the total lesions received prior EBRT with a median dose of $50.4 \mathrm{~Gy}$ in 2-8 fractions (IQR, 30-50.4). Chemotherapy was given to $82.1 \%$ of patients and $55.0 \%$ received neoadjuvant chemotherapy. Surgical status at diagnosis, deemed in a multidisciplinary case review, included resectable (entire cohort: $39.2 \%$, single fraction: $41.3 \%$, multifraction: $37.7 \%$ ), borderline resectable (entire cohort: $14.0 \%$, single fraction: $2.2 \%$, multifraction: $19.1 \%$ ), locally advanced/unresectable (entire cohort: 42.3\%, single fraction: $55.4 \%$, multifraction:37.2\%), and medically inoperable (entire cohort:4.5\%, single fraction: $1.1 \%$, multifraction: 6.0 ). Surgical resection was performed on $46.7,44.6$, and $47.7 \%$ of the entire cohort, single fraction patients, and multifraction patients, respectively.

\section{SBRT Treatment Characteristics}

Stereotactic body radiation therapy was delivered by Trilogy (41.2\%), Truebeam (34.1\%), or CyberKnife (24.7\%) in either one fraction $(30.9 \%)$ or multiple fractions (69.1\%). Median dose was $24 \mathrm{~Gy}$ (range 18-25) for single fraction and 36 Gy (range 24-36) for three fractions. One patient received $24 \mathrm{~Gy}$ in two fractions with all others receiving three-fraction regimens if treated in multiple fractions. For the entire cohort, GTV was $12.3 \mathrm{~cm}^{3}$ (IQR 8.6-21.3) and PTV was $17.9 \mathrm{~cm}^{3}$ (IQR 12-17). For patients who received neoadjuvant SBRT, the median time to surgery was 1.8 months (IQR 1.44-3.84). For those who received adjuvant SBRT, the median time from surgery was 3.77 months (IQR 2.17-12.15).

\section{Overall Survival}

At a median follow-up of 17.3 months (IQR 10.1-29.3 months), the median survival for the entire cohort was 17.8 months with a 2-year OS of 35.3\% (Table 2). Univariate analysis demonstrated superior 2-year survival was significantly associated with age $(p<0.001)$, pre-SBRT CA19-9 $(p<0.001)$, post-SBRT CA19-9 $(p=0.011)$, non-robotic treatment platform $(p=0.013)$, PTV volume $(p<0.001)$, recurrent lesions $(p=0.001)$, surgery $(p<0.001)$, and multifraction SBRT $(p=0.019)$. While univariate analysis showed multifraction schemes to have a higher 2-year OS $30.5 \%$ vs. $37.5 \%$, it did not hold significance on multivariate analysis. Only surgery $[p=<0.001$, HR 0.31 (95\% CI, 0.19-0.51)] and post-SBRT CA19-9 $[p=0.009$, HR 1.01 (95\% CI, 1.01-1.01)] maintained significance on multivariate analysis (Table 3). For patients receiving resection, 2-year OS was $59.8 \%$ compared to $14.3 \%$ for those not receiving surgical resection.

\section{Local Control}

Two-year LC was $66.1 \%$ for the entire cohort, $56.8 \%$ for single fraction, and $69.7 \%$ for multifraction SBRT (Figure 2). Univariate 
TABLE 1 | Patient characteristics

\begin{tabular}{lc}
\hline Characteristics & Value $(\boldsymbol{n}=\mathbf{2 9 1}$ lesions) \\
\hline Age (years, range) & $69(33-90)$ \\
Gender & \\
Female & $145(49.8 \%)$ \\
Male & $146(50.2 \%)$
\end{tabular}

\section{CA19-9 value (median value, IQR)}

$\begin{array}{lr}\text { At diagnosis } & 221(80-733) \\ \text { Pre-SBRT } & 81(21-378) \\ \text { Post-SBRT } & 73(23-342)\end{array}$

\section{SMAD4 mutated}

No

Yes

Unknown

$33(11.3 \%)$

$29(10 \%)$

$229(78.7 \%)$

\section{Surgical status}

Resectable entire cohort

Resectable single fraction

Resectable multifraction

Borderline resectable

Borderline resectable single fraction

Borderline resectable multifraction

Unresectable

Unresectable single fraction

Unresectable multifraction

Medically inoperable

Medically inoperable single fraction

Medically inoperable multifraction

\section{Surgery}

Yes entire cohort

Yes single fraction

Yes multifraction

No entire cohort

No single fraction

No multifraction

\section{Any chemotherapy}

Yes

No

Unknown

$112(39.2 \%)$

$38(41.3 \%)^{\mathrm{a}}$

$75(37.6 \%)^{b}$

$40(14.0 \%)$

$2(2.2 \%)^{\mathrm{a}}$

$38(19.1 \%)^{\mathrm{b}}$

$121(41.5 \%)$

$51(55.4 \%)^{\mathrm{a}}$

$74(37.2 \%)^{b}$

$13(4.5 \%)$

$1(1.1 \%)^{\mathrm{a}}$

$12(6.0 \%)^{\mathrm{b}}$

\section{Neoadjuvant chemotherapy \\ Yes \\ No \\ Unknown}

136 (46.7\%)

$41(44.6 \%)^{a}$

$95(47.7 \%)^{b}$

155 (53.3\%)

$51(55.4 \%)^{\mathrm{a}}$

$105(52.3 \%)^{\mathrm{b}}$

\section{Location}

Body

Heal

Tail

Uncinate

Neck

Genu

Multiple

\section{Prior EBRT}

Yes

No

Previous EBRT dose (median, IQR)

\section{Treatment platform}

Trilogy

CyberKnife

Truebeam

\section{Recurrent lesion}

Yes

239 (82.1\%)

$42(14.4 \%)$

$10(3.4 \%)$

$160(55 \%)$

$115(39.5 \%)$

$16(5.5 \%)$

$32(11 \%)$

$183(62.9 \%)$

$8(2.7 \%)$

$22(7.6 \%)$

$14(4.8 \%)$

$1(0.3 \%)$

$31(10.7 \%)$

38 (13.1\%)

253 (86.9\%)

$50.4(30-50.4)$

120 (41.2\%)

$72(24.7 \%)$

99 (34\%)

39 (13.4\%)
TABLE 1 | Continued

\begin{tabular}{lc}
\hline Characteristics & Value $(\boldsymbol{n}=\mathbf{2 9 1}$ lesions) \\
\hline No & $252(86.6 \%)$ \\
\hline GTV $\left(\mathrm{cm}^{3}\right)$ (median, IQR) & $12.3(8.6-21.3)$ \\
PTV $\left(\mathbf{c m}^{3}\right)$ (median, IQR) & $17.9(12-27)$ \\
\hline
\end{tabular}

Fractionation

Single $\quad 90(30.9 \%)$

Multifraction

$201(69.1 \%$

Dose

18 Gy in 1 fraction $\quad 2(0.7 \%)$

20 Gy in 1 fraction $\quad 4(1.4 \%)$

20 Gy in 2 fractions $\quad 1(0.3 \%)$

22 Gy in 1 fraction $\quad 17(5.8 \%)$

24 Gy in 1 fraction $\quad 62(21.3 \%)$

24 Gy in 2 fraction $\quad 1(0.3 \%)$

24 Gy in 3 fraction $\quad 2(0.7 \%)$

25 Gy in 1 fraction $\quad 5(1.7 \%)$

27 Gy in 3 fractions $\quad 7(2.4 \%)$

30 Gy in 3 fractions $\quad 28(9.6 \%)$

36 Gy in 3 fractions $\quad 162(55.7 \%)$

\section{BED $_{10 \text { Gy }}$}

Median (IQ range)

$180 \mathrm{~Gy}_{10}(180-183.3)$

aPercentages out of the 92 patients who received single-fraction SBRT.

${ }^{b}$ Percentages out of the 199 patients who received multifraction SBRT.

SBRT, stereotactic body radiation therapy.

analysis demonstrated superior 2-year LC significantly associated with treatment platform $(p=0.002)$, non-recurrent lesions $(p<0.001)$, surgery $(p=0.01)$, and multifraction SBRT $(p=0.004)$. Two-year LC for patients who received surgical resection was 74.1 vs. $53.9 \%$ for those who did not. On multivariate analysis, multifractionation $[p=0.009$, HR 0.53 (95\% CI, $0.33-0.85)$ ] was associated with higher LC, whereas recurrent lesions led to lower LC [ $p=0.003$, HR 2.31 (95\% CI, 1.32-4.05)] (Table 4). Two-year LC for recurrent lesions was $35.4 \%$ compared to $70.4 \%$ for non-recurrent lesions.

\section{RC and Distant Metastases}

One- and two-year RC rates were 89.2 and $86.3 \%$, respectively. A higher post-SBRT CA19-9 was the only variable found to be significantly associated with inferior RC on univariate and multivariate analysis $[p=0.02$, HR 1.01 (95\% CI, 1.01-1.01)] (Table 5). At one and two years, the Kaplan-Meier estimated rate of DM was 39.5 and 56.4\%, respectively. Univariate analysis identified CA19-9 at diagnosis $(p=0.041)$, pre-SBRT CA 19-9 $(p=0.001)$, and post-SBRT CA $19-9(p=0.006)$ associated with increased distant metastases. Post-SBRT CA19-9 was the only variable to maintain significance on multivariate analysis [ $p=0.001$, HR 1.01 (95\% CI, 1.01-1.01)] (Table 5). Treatment fractionation was not found to be associated with either RC or distant metastases.

\section{Late Radiation Toxicity}

For the entire cohort, the Kaplan-Meier estimated advanced grade $3+$ toxicity rate at 1 - and 2-years was $2.5 \%$ (95\% CI, $2.3-2.7 \%)$ advanced grade. No significant difference was noted based on single- or multifraction use (2-year advanced grade $3+$ 
toxicity estimate 2.3 vs. $2.8 \%, p=0.747)$. Prior radiation therapy was the only factor predictive of advanced grade 3+ toxicity [ $p=0.019$, HR 4.58 (95\% CI, 1.29-16.21)] (Table 6). For patients who received surgery, advanced grade $3+$ toxicity rate at 2 -years was $1.8 \%$ compared to $3.2 \%$ for non-surgical patients $(p=0.258)$. Three patients experienced grade 4 toxicities which included an ileal obstruction $(n=1)$, obstruction gastric $(n=1)$, and duodenal stenosis $(n=1)$ all requiring urgent operative intervention. Eight patients experienced grade 3 toxicities which included nausea $(n=3)$, enteritis $(n=2)$, enterocolitis $(n=1)$, ileal hemorrhage $(n=1)$, and biliary tract infection $(n=1)$.

\section{DISCUSSION}

This retrospective review aimed to assess the role SBRT fractionation on OS and LC of pancreatic adenocarcinoma while also determining the rates of late radiation toxicity for this treatment modality. This study identified three-fraction SBRT

TABLE 2 | Univariate and multivariate analyses for overall survival (OS).

\begin{tabular}{|c|c|c|}
\hline Variable & 2-year OS & $p$ Value \\
\hline Age & - & $<0.001$ \\
\hline \multicolumn{3}{|l|}{ CA19-9 } \\
\hline At diagnosis & - & 0.604 \\
\hline Pre-SBRT & - & $<0.001$ \\
\hline By median ( $\leq 83$ vs. <83) & - & 0.457 \\
\hline$\leq 90$ vs. $>90$ & - & 0.347 \\
\hline Post-SBRT & - & 0.011 \\
\hline By median ( $\leq 73$ vs. $>73$ ) & 54.1 vs. $26.4 \%$ & $<0.001$ \\
\hline$\leq 90$ vs. $>90$ & 52.1 vs. $25.5 \%$ & $<0.001$ \\
\hline SMAD4 mutation & & 0.968 \\
\hline Location & - & 0.283 \\
\hline Prior EBRT & - & 0.241 \\
\hline \multicolumn{3}{|l|}{ Treatment platform } \\
\hline All (Trilogy, Truebeam, CK) & 39.5 vs. 27.8 vs. $35.9 \%$ & 0.036 \\
\hline $\begin{array}{l}\text { Non-robotic (T/T) vs. CyberKnife } \\
\text { All (Trilogy, Truebeam, CK) }\end{array}$ & 37.9 vs. $27.8 \%$ & 0.013 \\
\hline \multicolumn{3}{|l|}{ Recurrent lesion } \\
\hline Recurrent vs. not & 69.4 vs. $30.4 \%$ & 0.001 \\
\hline Surgery (yes vs. no) & 59.8 vs. $14.3 \%$ & $<0.001$ \\
\hline \multicolumn{3}{|l|}{ Dosimetry } \\
\hline GTV volume & - & 0.399 \\
\hline GTV max dose & - & 0.163 \\
\hline GTV min dose & - & 0.793 \\
\hline PTV volume & - & $<0.001$ \\
\hline By median ( $\leq 18$ vs. $>18 \mathrm{~cm}^{3}$ ) & 44 vs. $31.9 \%$ & 0.033 \\
\hline PTV max dose & - & 0.847 \\
\hline PTV min dose & - & 0.529 \\
\hline PTV mean dose & - & 0.982 \\
\hline Small bowel max dose & - & 0.365 \\
\hline Small bowel mean dose & - & 0.179 \\
\hline Single vs. multifraction & 30.5 vs. $37.5 \%$ & 0.019 \\
\hline $\mathrm{BED}_{10}$ Gy & - & 0.151 \\
\hline Variable & HR (95\% Cl) & $p$ Value \\
\hline Post-SBRT CA 19-9 (continuous) & $1.01(1.01-1.01)$ & 0.009 \\
\hline \multicolumn{3}{|l|}{ Surgery } \\
\hline Not completed & 1.00 (reference) & - \\
\hline Completed & $0.31(0.19-0.50)$ & $<0.001$ \\
\hline
\end{tabular}

Boldface values are significant predictors ( $p$ value $<0.05$ ). provides superior 2-year LC over single fractionation, but may not be associated with improved OS. Additionally, treatment with either single- or multifraction SBRT was delivered with reasonable levels of toxicity with prior radiation therapy most predictive of late radiation toxicities. Consistent with numerous other reports, these data also support surgery as the most important factor for OS (24).

Pollom et al. previously reported on 167 patients with unresectable pancreatic adenocarcinoma comparing single- versus five-fraction SBRT. Median OS for all patients was 13.6 months with 12-month survival from SBRT at 30.8 and $34.9 \%$ for single- and multifraction, respectively. The 12-month cumulative incidence rates of local recurrence were 9.5 and $11.7 \%$ for single and multifraction, respectively. Neither OS from SBRT nor local recurrence demonstrated significant difference between singleand multifraction groups. Cumulative GI grade $2+$ toxicity by 12 months was $26.1 \%$ for single fraction and $7.8 \%$ for multifraction which was a significant difference (20). In contrast, our data show a significant improvement in LC with multifraction as compared to single-fraction regimens. This difference is most likely due to the

TABLE 3 | Univariate and multivariate analysis for local control (LC).

\begin{tabular}{lcc}
\hline Variable & 2-year LC & $\boldsymbol{p}$ Value \\
\hline Age & - & 0.549 \\
\hline CA 19-9 & & \\
At diagnosis & - & 0.581 \\
Pre-SBRT & - & 0.820 \\
Post-SBRT & - & 0.082 \\
SMAD4 mutation & - & 0.791 \\
Location & - & 0.427 \\
Prior EBRT & - & 0.586 \\
\hline
\end{tabular}

Treatment platform

All (Trilogy, Truebeam, CK)

Non-robotic (T/T) vs. CyberKnife

73.7 vs. 61.7 vs. $58.5 \% \quad \mathbf{0 . 0 2 2}$ 68.2 vs. $58.5 \% \quad \mathbf{0 . 0 1 0}$

All (Trilogy, Truebeam, CK)

\section{Recurrent lesion}

Recurrent vs. not

Surgery (yes vs. no)

35.4 vs. $70.4 \%$ 74.1 vs. $53.9 \%$

$<0.001$

Dosimetry

GTV volume

GTV max dose

GTV min dose

PTV volume

PTV max dose

PTV min dose

PTV mean dose

Small bowel max dose

Small bowel mean dose

Single vs. multifraction

BED $_{10 \text { gy }}$ (continuous)

\begin{tabular}{lll}
\hline Variable HR $(95 \% \mathrm{Cl})$ & $p$ Value
\end{tabular}

\section{Recurrent lesion}

$\begin{array}{lll}\text { No } & 1.00 \text { (reference) } & \mathbf{0 . 0 0 3}\end{array}$

Yes

$2.31(1.32-4.05)$

Fractionation scheme

Single fraction $\quad 1.00$ (reference)

$0.53(0.33-0.85)$

Multifraction

$0.53(0.33-0$ 


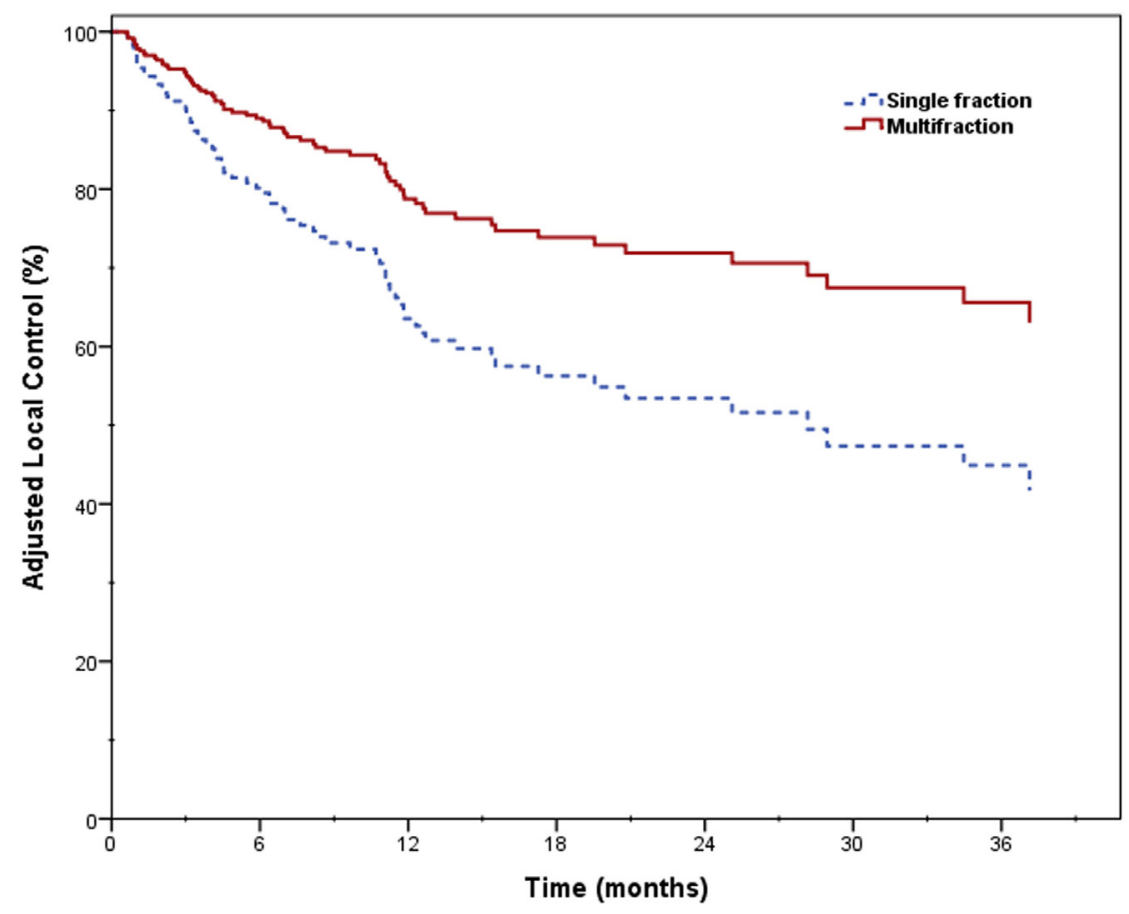

FIGURE 2 | Local control for single- and multifraction stereotactic body radiation therapy.

significantly larger cohort used in the present study. Additional differences that may have contributed included our study reporting on all pancreatic adenocarcinoma except those with distant metastases as opposed to only unresectable tumors including those with distant metastases at treatment. Additionally, our multifraction group consisted of $36 \mathrm{~Gy}$ in three fractions as opposed to $33 \mathrm{~Gy}$ in five fractions. Finally, because the linear quadratic model in not applicable for hypofractionated treatments, there could be a difference in BED between these two regimens which is currently unknown.

We believe our difference observed in LC between single and multifraction SBRT is most likely a result of differences in tumor dose. In our initial experience with single fraction SBRT there was significant concern for bowel toxicity. In an effort to reduce toxicity, dose to the small bowel was limited therefore compromising PTV coverage. Multifraction regimens allowed us to be less conservative with bowel dosing and therefore allow for greater coverage of the tumor. This would further explain why we did not observe a difference in advanced grade $3+$ toxicity between these regimens as demonstrated by Pollom et al. These two studies seem to demonstrate the trade-off between toxicity and LC for single fraction SBRT. Our observed difference in LC however could also be a result of patient demographics independent of fractionation. As reports of increased toxicity with single fraction surfaced, our institution slowly switched to multifraction regimens. This lead a greater proportion of patients treated in later years received three-fraction SBRT. It is possible this observed difference was a result of differences in chemotherapy; however, chemotherapy regimens were poorly reported and demonstrated significant heterogeneity between and within single- and multifraction cohorts. Additionally, there was higher proportion of recurrent disease in those treated with single fraction SBRT. As we have shown, patients with recurrent disease demonstrated worse LC which may have affected the results observed in single-fraction patients. Although surgical resection has been shown to be the most significant predictive factor for clinical outcomes, it is unlikely playing a role in the observed difference between different fraction regimens as resection rates were similar between both groups. SBRT has also been an enticing modality in the treatment of locally advanced and borderline resectable pancreatic adenocarcinoma due to its improved rates of LC and increased rates of resection in these tumors (25). Although previous trials analyzing single fraction SBRT demonstrated excellent LC, they were associated with unacceptable rates of toxicity $(18,19)$. This leads to the adoption of multifraction regimens. Chuong et al. reported on 73 patients with borderline resectable (78.1\%) and locally advanced (21.9\%) pancreatic adenocarcinoma treated with induction chemotherapy followed by five-fraction SBRT. Borderline resectable and locally advanced tumors experienced a median OS of 16.4 and 15 months and a 1-year progression free survival of 42.8 and $41 \%$, respectively, with $5.3 \%$ advanced grade $3+$ toxicity (10). Mahadevan et al. reported on 36 patients with nonmetastatic locally advanced pancreatic cancer treated with three-fraction SBRT with a total dose of 24, 30, or 36 Gy followed by gemcitabine therapy. Median OS was 14.3 months; LC was $78 \%$ with a median progression-free survival of 9.6 months and $5.5 \%(n=2)$ developed advanced grade $3+$ toxicity. Although previous reports identified unacceptable rates of advanced toxicity with single-fraction SBRT our results demonstrated no significant difference between single- and multifractionation on advanced toxicity, which may reflect variances in volumes, constraints and total dose between institutions. Additionally, as previously stated, due to early reports of high rates of late GI toxicity, our institution limited dose to the small 
TABLE 4 | Univariate and multivariate analysis for regional control (RC).

\begin{tabular}{|c|c|c|}
\hline Variable & 2-year RC & $p$ Value \\
\hline Age & - & 0.271 \\
\hline \multicolumn{3}{|l|}{ CA 19-9 } \\
\hline At diagnosis & - & 0.535 \\
\hline Pre-SBRT & - & 0.413 \\
\hline Post-SBRT & - & 0.020 \\
\hline By median ( $\leq 73$ vs. $>73$ ) & - & 0.580 \\
\hline$\leq 90$ vs. $>90$ & - & 0.891 \\
\hline SMAD4 mutation & - & 0.677 \\
\hline Location & - & 0.942 \\
\hline Prior EBRT & - & 0.677 \\
\hline \multicolumn{3}{|l|}{ Treatment platform } \\
\hline All (Trilogy, Truebeam, CK) & - & 0.735 \\
\hline Non-robotic (T/T) vs. CyberKnife & - & 0.725 \\
\hline \multicolumn{3}{|l|}{ Recurrent lesion } \\
\hline Recurrent vs. not & - & 0.137 \\
\hline Surgery (yes vs. no) & - & 0.434 \\
\hline \multicolumn{3}{|l|}{ Dosimetry } \\
\hline GTV volume & - & 0.685 \\
\hline GTV max dose & - & 0.904 \\
\hline GTV min dose & - & 0.761 \\
\hline PTV volume & - & 0.752 \\
\hline PTV max dose & - & 0.958 \\
\hline PTV min dose & - & 0.608 \\
\hline PTV mean dose & - & 0.789 \\
\hline Small bowel max dose & - & 0.748 \\
\hline Small bowel mean dose & - & 0.600 \\
\hline Single vs. multifraction & - & 0.541 \\
\hline $\mathrm{BED}_{10 \text { Gy }}$ (continuous) & - & 0.067 \\
\hline Variable & HR (95\% Cl) & $p$ Value \\
\hline Post-SBRT CA 19-9 & $1.01(1.01-1.01)$ & 0.020 \\
\hline
\end{tabular}

Boldface values are significant predictors ( $p$ value $<0.05$ ).

bowel in an attempt to minimize toxicity rates. Our lack of ability to identify differences in toxicity could also be secondary to the inherent limitation of retrospective studies.

Stereotactic body radiation therapy has also been identified as an effective treatment modality in treatment of recurrent lesions following resection. Dagolglu et al. reported on 30 patients treated with SBRT for recurrent pancreatic cancer with prior radiation therapy. Patients received a median of $25 \mathrm{~Gy}$ in five fractions. Median OS was 14 months, 2-year LC was 78, and 7\% advanced grade $3+$ toxicity (26). Here, we reported significantly greater risk of advanced toxicity with prior radiation therapy while Dagolglu et al. demonstrated reasonable toxicity in patients with prior radiation. The lower toxicity seen with Dagolglu et al. could be secondary to the five fraction regimen used as opposed to the three fractions or one fraction mostly used in our study. Also, $25 \mathrm{~Gy}$ in five fractions has a lower biological effective dose than our regimens, which may compromise LC.

Herman et al. recently reported on a multi-institutional study combining Gemcitabine with five-fraction SBRT in patients with locally advanced pancreatic cancer (27). Forty-nine patients with locally advanced patients were treated with up to three doses of Gemcitabine $\left(1,000 \mathrm{mg} / \mathrm{m}^{2}\right)$ followed by a 1-week break and then SBRT, 33 Gy in five fractions over 1-2 weeks. After SBRT, patients were continued on Gemcitabine until progression. Median OS was 14 months and 2-year OS was $18 \%$. Freedom from local
TABLE 5 | Univariate and multivariate analysis for distant metastasis (DM).

\begin{tabular}{|c|c|c|}
\hline Variable & 2-year DM & $p$ Value \\
\hline Age & - & 0.348 \\
\hline \multicolumn{3}{|l|}{ CA 19-9 } \\
\hline At diagnosis & - & 0.041 \\
\hline Pre-SBRT & - & 0.001 \\
\hline By median ( $\leq 83$ vs. >83) & 48.9 vs. $59.8 \%$ & 0.039 \\
\hline$\leq 90$ vs. $>90$ & 50.3 vs. $59.2 \%$ & 0.054 \\
\hline Post-SBRT & - & 0.006 \\
\hline By median ( $\leq 73$ vs. $>73$ ) & 41.8 vs. $70.8 \%$ & 0.004 \\
\hline$\leq 90$ vs. $>90$ & 47.7 vs. $66.6 \%$ & 0.067 \\
\hline SMAD4 mutation & - & 0.696 \\
\hline Location & - & 0.745 \\
\hline Prior EBRT & - & 0.815 \\
\hline \multicolumn{3}{|l|}{ Treatment platform } \\
\hline All (Trilogy, Truebeam, CK) & - & 0.467 \\
\hline Non-robotic (T/T) vs. CyberKnife & - & 0.163 \\
\hline \multicolumn{3}{|l|}{ Recurrent lesion } \\
\hline Recurrent vs. not & - & 0.586 \\
\hline Surgery (yes vs. no) & - & 0.124 \\
\hline \multicolumn{3}{|l|}{ Dosimetry } \\
\hline GTV volume & - & 0.664 \\
\hline GTV max dose & - & 0.380 \\
\hline GTV min dose & - & 0.694 \\
\hline PTV volume & - & 0.564 \\
\hline PTV max dose & - & 0.480 \\
\hline PTV min dose & - & 0.176 \\
\hline PTV mean dose & - & 0.900 \\
\hline Small bowel max dose & - & 0.487 \\
\hline Small bowel mean dose & - & 0.499 \\
\hline Single vs. multifraction & - & 0.226 \\
\hline BED $_{10}$ Gy (continuous) & - & 0.429 \\
\hline Variable & HR (95\% Cl) & $p$ Value \\
\hline Post-SBRT CA 19-9 & $1.01(1.01-1.01)$ & 0.001 \\
\hline
\end{tabular}

Boldface values are significant predictors ( $p$ value $<0.05$ ).

progression at 1 -year was $78 \%$. On multivariate analysis PET positive disease prior to SBRT and CA19-9 > 90 after SBRT were associated with an increased risk of death. Acute grade 2 plus toxicity included enteritis, gastritis and ulcer. There was 1 grade 4 toxicity of a duodenal fistula. There was $11 \%$ advanced grade 2 or greater toxicity, again mostly enteritis, gastritis, and ulcer. Their advanced toxicity was low like ours showing feasibility of multifraction regimens.

We showed higher post-SBRT CA 19-9 were associated with lower survival and higher rates of regional and DM. This is similar to the results reported by Herman et al, who showed post-SBRT values greater than 90 were associated with a lower survival on multivariate analysis (27). These results of post-SBRT CA19-9 associated with survival but not CA19-9 at diagnosis or pre-SBRT indicate posttreatment CA19-9 as a surrogate for treatment efficacy. Similar data have been reported in the literature identifying a decrease in CA19-9 following treatment to be a predictor of OS (28-30). Future trials evaluating the role of SBRT for pancreatic carcinoma should consider evaluating the affect SBRT has on CA19-9 values.

This study adds to the current growing body of literature that demonstrates the effectiveness and tolerability of SBRT for pancreatic adenocarcinoma. Following the initial reports demonstrating unacceptably high rates of advanced GI toxicity with single fraction SBRT, many institutions transitioned to multifraction regimens. 
TABLE 6 | Kaplan-Meier estimates for various end points.

\begin{tabular}{|c|c|c|}
\hline End point & $\begin{array}{c}\text { Kaplan-Meier } \\
\text { estimate ( } 95 \% \\
\text { confidence interval) }\end{array}$ & \\
\hline \multicolumn{3}{|l|}{ Median follow-up } \\
\hline From SBRT (months) (IQ range) & $17.3(10.1-29.3)$ & \\
\hline \multicolumn{3}{|l|}{ Median survival } \\
\hline Median survival (months) (95\% Cl) & $17.8(15.7-20.0)$ & \\
\hline 12 months (95\% Cl) & $69.7(64.4-75.0)$ & \\
\hline 24 months $(95 \% \mathrm{Cl})$ & $35.3(29.8-40.9)$ & \\
\hline \multicolumn{3}{|l|}{ Local control from SBRT } \\
\hline 12 months $(95 \% \mathrm{Cl})$ & $73.7(67.6-79.8)$ & \\
\hline 24 months $(95 \% \mathrm{Cl})$ & $66.1(58.5-73.4)$ & \\
\hline \multicolumn{3}{|l|}{ Regional control from SBRT } \\
\hline 12 months $(95 \% \mathrm{Cl})$ & $89.2(84.9-93.5)$ & \\
\hline 24 months $(95 \% \mathrm{Cl})$ & $86.3(81.0-91.6)$ & \\
\hline \multicolumn{3}{|l|}{ Distant metastases from SBRT } \\
\hline 12 months $(95 \% \mathrm{Cl})$ & 39.5 (33.0-46.0) & \\
\hline 24 months $(95 \% \mathrm{Cl})$ & $56.4(48.6-64.2)$ & \\
\hline \multicolumn{3}{|l|}{ Toxicity } \\
\hline \multicolumn{3}{|l|}{ Grade $2+$ toxicity } \\
\hline 1-year toxicity (95\% Cl) & $7.8(4.7-10.9)$ & \\
\hline 2-year toxicity (95\% Cl) & $7.8(4.7-10.9)$ & \\
\hline \multicolumn{3}{|l|}{ Grade 3+ toxicity } \\
\hline 1-year toxicity (95\% Cl) & $2.5(2.3-2.7)$ & \\
\hline 2-year toxicity $(95 \% \mathrm{Cl})$ & $2.5(2.3-2.7)$ & \\
\hline \multicolumn{3}{|c|}{ Univariable analysis for advanced toxicities } \\
\hline Variable (grade 2+advanced toxicity) & HR $(95 \% \mathrm{Cl})$ & $p$ Value \\
\hline Prior radiotherapy & $3.26(1.33-8.00)$ & 0.010 \\
\hline GTV volume & - & 0.201 \\
\hline PTV volume & - & 0.736 \\
\hline Treatment platform & - & 0.57 \\
\hline Single vs. multifraction & - & 0.737 \\
\hline Small bowel max dose & - & 0.955 \\
\hline Small bowel mean dose & - & 0.774 \\
\hline Variable (grade $3+$ advanced toxicity) & $\mathrm{HR}(95 \% \mathrm{Cl})$ & $p$ Value \\
\hline Prior radiotherapy & $4.58(1.29-16.21)$ & 0.019 \\
\hline GTV volume & - & 0.396 \\
\hline PTV volume & - & 0.997 \\
\hline Treatment platform & - & 0.427 \\
\hline Single vs. multifraction & - & 0.983 \\
\hline Small bowel max dose & - & 0.899 \\
\hline Small bowel mean dose & - & 0.795 \\
\hline
\end{tabular}

Despite previous reports indicating multifraction SBRT produced decreased rates of toxicity, it was unclear whether this came at the cost of other clinical outcomes. Through analyzing the largest cohort comparing single to multifraction SBRT, we identified multifraction treatment schedules to be associated with improved LC while reinforcing the significant role surgery and CA19-9 levels play in prognosis $(2,18,31-34)$. These results support the continued use of multifraction regimens. Taken together with prior work, multifraction SBRT appears to provide either improved LC or reduced rates of toxicity as compared to single fraction (20).

Despite the above study showing a significant difference in LC between SBRT fractionation, there were numerous limitations to this work. Our cohort represented a very heterogeneous population as it included resected and unresected disease, recurrent disease, as well as patients with prior EBRT. Additionally, as this was a retrospective review the breakdown of patients within oneand three-fraction SBRT were not matched. Another limitation was our inability to capture detailed chemotherapy data on these patients. As patients with single fraction were treated in earlier years it is possible there is an unseen effect of advancements in chemotherapy over time. Regarding toxicity, although we report very low rates, this may be artificially low due to uncaptured toxicity associated with retrospective reports. However, our low toxicity rates are possibly secondary to our institution editing the PTV out of the bowel to reduce toxicity. Finally, CA19-9 levels and SMAD4 mutation status were not obtained in all patients, likely due to limited testing. Prospective studies will be needed to provide a more rigorous analysis of the role of SBRT fractionation in pancreatic adenocarcinoma as well as the possible contribution of SMAD4 mutation status and evolving systemic therapy on LC.

\section{CONCLUSION}

This single institution retrospective review of 291 patients with pancreatic adenocarcinoma identified multifraction regimens SBRT had a higher LC than single fractionation regimens. Although multifraction regimens displayed a higher OS on univariate analysis it did not hold significance on MVA. Post-SBRT CA19-9 was found to be significant factor for OS, RC, and DM. Finally, we showed low rates of advanced grade $2+$ and grade $3+$ toxicity associated with SBRT. This single institution report is the largest retrospective series showing multifraction regimens SBRT is associated with a higher LC than single fractionation regimens.

\section{ETHICS STATEMENT}

The following study was approved by the University of Pittsburgh Institutional Review Board.

\section{AUTHOR CONTRIBUTIONS}

PS: collected data from electronic medical records and paper records; wrote and revised manuscript; and approves final version of manuscript. MB: conceived and designed the project; assisted with data collection; revised the manuscript multiple times; and approves final version of manuscript. BG: performed statistical analysis on collected data; revised the manuscript; and approves final version of manuscript. $\mathrm{KH}$ : collected data regarding SMAD4 mutation status; provided revisions to manuscript; and approves final version of manuscript. KQ: aided in the interpretation of results as they related to radiation oncology; provided revisions to manuscript; and approves final version of manuscript. NB: aided in the interpretation of results as they relate to medical oncology; provided revisions to manuscript; and approves final version of manuscript. SB: aided in the interpretation of results as they relate to radiation oncology; provided revisions to manuscript; and approves final version of manuscript. HZ: aided in the interpretation of results as they relate to surgical oncology; provided revisions to manuscript; approves final version of manuscript. $\mathrm{DH}$ : conceived and designed project with $\mathrm{MB}$; provided multiple revisions to manuscript; and approves final version of manuscript. 


\section{REFERENCES}

1. Siegel RL, Miller KD, Jemal A. Cancer statistics, 2016. CA Cancer J Clin (2016) 66:7-30. doi:10.3322/caac. 21332

2. Ansari D, Gustafsson A, Andersson R. Update on the management of pancreatic cancer: surgery is not enough. World J Gastroenterol (2015) 21:3157-65. doi:10.3748/wjg.v21.i11.3157

3. Ryan DP, Hong TS, Bardeesy N. Pancreatic adenocarcinoma. N Engl J Med (2014) 371:1039-49. doi:10.1056/NEJMra1404198

4. Moningi S, Dholakia AS, Raman SP, Blackford A, Cameron JL, Le DT, et al. The role of stereotactic body radiation therapy for pancreatic cancer: a single-institution experience. Ann Surg Oncol (2015) 22:2352-8. doi:10.1245/ s10434-014-4274-5

5. Mahadevan A, Jain S, Goldstein M, Miksad R, Pleskow D, Sawhney M, et al. Stereotactic body radiotherapy and gemcitabine for locally advanced pancreatic cancer. Int J Radiat Oncol Biol Phys (2010) 78:735-42. doi:10.1016/j. ijrobp.2009.08.046

6. Dilling TJ, Hoffe SE. Stereotactic body radiation therapy: transcending the conventional to improve outcomes. Cancer Control (2008) 15:104-11. doi:10.1177/107327480801500202

7. Trakul N, Koong AC, Chang DT. Stereotactic body radiotherapy in the treatment of pancreatic cancer. Semin Radiat Oncol (2014) 24:140-7. doi:10.1016/j. semradonc.2013.11.008

8. Rubio C, Morera R, Hernando O, Leroy T, Lartigau SE. Extracranial stereotactic body radiotherapy. Review of main SBRT features and indications in primary tumors. Rep Pract Oncol Radiother (2013) 18:387-96. doi:10.1016/j. rpor.2013.09.009

9. Rwigema JC, Parikh SD, Heron DE, Howell M, Zeh H, Moser AJ, et al. Stereotactic body radiotherapy in the treatment of advanced adenocarcinoma of the pancreas. Am J Clin Oncol (2011) 34:63-9. doi:10.1097/ COC.0b013e3181d270b4

10. Chuong MD, Springett GM, Freilich JM, Park CK, Weber JM, Mellon EA, et al. Stereotactic body radiation therapy for locally advanced and borderline resectable pancreatic cancer is effective and well tolerated. Int J Radiat Oncol Biol Phys (2013) 86:516-22. doi:10.1016/j.ijrobp.2013.02.022

11. Chang DT, Schellenberg D, Shen J, Kim J, Goodman KA, Fisher GA, et al. Stereotactic radiotherapy for unresectable adenocarcinoma of the pancreas. Cancer (2009) 115:665-72. doi:10.1002/cncr.24059

12. Comito T, Cozzi L, Zerbi A, Franzese C, Clerici E, Tozzi A, et al. Clinical results of stereotactic body radiotherapy (SBRT) in the treatment of isolated local recurrence of pancreatic cancer after R0 surgery: a retrospective study. Eur J Surg Oncol (2017) 43(4):735-42. doi:10.1016/j.ejso.2016.12.012

13. Goyal K, Einstein D, Ibarra RA, Yao M, Kunos C, Ellis R, et al. Stereotactic body radiation therapy for nonresectable tumors of the pancreas. J Surg Res (2012) 174:319-25. doi:10.1016/j.jss.2011.07.044

14. Lominska CE, Unger K, Nasr NM, Haddad N, Gagnon G. Stereotactic body radiation therapy for reirradiation of localized adenocarcinoma of the pancreas. Radiat Oncol (2012) 7:74. doi:10.1186/1748-717X-7-74

15. Brunner TB, Nestle U, Grosu AL, Partridge M. SBRT in pancreatic cancer: what is the therapeutic window? Radiother Oncol (2015) 114:109-16. doi:10.1016/j. radonc.2014.10.015

16. Bijlani A, Aguzzi G, Schaal DW, Romanelli P. Stereotactic radiosurgery and stereotactic body radiation therapy cost-effectiveness results. Front Oncol (2013) 3:77. doi:10.3389/fonc.2013.00077

17. Kim SK, Wu CC, Horowitz DP. Stereotactic body radiotherapy for the pancreas: a critical review for the medical oncologist. J Gastrointest Oncol (2016) 7:479-86. doi:10.21037/jgo.2015.10.01

18. Schellenberg D, Goodman KA, Lee F, Chang S, Kuo T, Ford JM, et al. Gemcitabine chemotherapy and single-fraction stereotactic body radiotherapy for locally advanced pancreatic cancer. Int J Radiat Oncol Biol Phys (2008) 72:678-86. doi:10.1016/j.ijrobp.2008.01.051

19. Schellenberg D, Kim J, Christman-Skieller C, Chun CL, Columbo LA, Ford JM, et al. Single-fraction stereotactic body radiation therapy and sequential gemcitabine for the treatment of locally advanced pancreatic cancer. Int J Radiat Oncol Biol Phys (2011) 81:181-8. doi:10.1016/j.ijrobp.2010. 05.006

20. Pollom EL, Alagappan M, von Eyben R, Kunz PL, Fisher GA, Ford JA, et al. Single- versus multifraction stereotactic body radiation therapy for pancreatic adenocarcinoma: outcomes and toxicity. Int J Radiat Oncol Biol Phys (2014) 90:918-25. doi:10.1016/j.ijrobp.2014.06.066

21. Eisenhauer EA, Therasse P, Bogaerts J, Schwartz LH, Sargent D, Ford R, et al. New response evaluation criteria in solid tumours: revised RECIST guideline (version 1.1). Eur J Cancer England: Oxford (2009) 45:228-47. doi:10.1016/j.ejca.2008.10.026

22. Yamada S, Fujii T, Hirakawa A, Kanda M, Sugimoto H, Kodera Y. Lymph node ratio as parameter of regional lymph node involvement in pancreatic cancer. Langenbecks Arch Surg (2016) 401:1143-52. doi:10.1007/s00423-016-1412-5

23. Isaji S, Kawarada Y, Uemoto S. Classification of pancreatic cancer: comparison of Japanese and UICC classifications. Pancreas (2004) 28:231-4. doi:10.1097/00006676-200404000-00003

24. Myrehaug S, Sahgal A, Russo SM, Lo SS, Rosati LM, Mayr NA, et al. Stereotactic body radiotherapy for pancreatic cancer: recent progress and future directions. Expert Rev Anticancer Ther (2016) 16:523-30. doi:10.1586/ 14737140.2016.1168698

25. Mellon EA, Hoffe SE, Springett GM, Frakes JM, Strom TJ, Hodul PJ, et al. Long-term outcomes of induction chemotherapy and neoadjuvant stereotactic body radiotherapy for borderline resectable and locally advanced pancreatic adenocarcinoma. Acta Oncol (2015) 54:979-85. doi:10.3109/028 4186X.2015.1004367

26. Dagoglu N, Callery M, Moser J, Tseng J, Kent T, Bullock A, et al. Stereotactic body radiotherapy (SBRT) reirradiation for recurrent pancreas cancer. J Cancer (2016) 7:283-8. doi:10.7150/jca.13295

27. Herman JM, Chang DT, Goodman KA, Dholakia AS, Raman SP, HackerPrietz A, et al. Phase 2 multi-institutional trial evaluating gemcitabine and stereotactic body radiotherapy for patients with locally advanced unresectable pancreatic adenocarcinoma. Cancer (2015) 121:1128-37. doi:10.1002/ cncr.29161

28. Chiorean EG, Von Hoff DD, Reni M, Arena FP, Infante JR, Bathini VG, et al. CA19-9 decrease at 8 weeks as a predictor of overall survival in a randomized phase III trial (MPACT) of weekly nab-paclitaxel plus gemcitabine versus gemcitabine alone in patients with metastatic pancreatic cancer. Ann Oncol (2016) 27:654-60. doi:10.1093/annonc/mdw006

29. Tzeng CW, Balachandran A, Ahmad M, Lee JE, Krishnan S, Wang H, et al. Serum carbohydrate antigen 19-9 represents a marker of response to neoadjuvant therapy in patients with borderline resectable pancreatic cancer. $H P B$ (Oxford) (2014) 16:430-8. doi:10.1111/hpb.12154

30. An X, Li YH, Lin XB, Wang FH, Feng F, Xu RH, et al. [Prognostic value of serum CA19-9 in patients with advanced pancreatic cancer receiving gemcitabine based chemotherapy]. Ai Zheng (2009) 28:286-91.

31. Kondo N, Murakami Y, Uemura K, Sudo T, Hashimoto Y, Sasaki H, et al. Elevated perioperative serum CA 19-9 levels are independent predictors of poor survival in patients with resectable cholangiocarcinoma. J Surg Oncol (2014) 110:422-9. doi:10.1002/jso.23666

32. Ziske C, Schlie C, Gorschlüter M, Glasmacher A, Mey U, Strehl J, et al. Prognostic value of CA 19-9 levels in patients with inoperable adenocarcinoma of the pancreas treated with gemcitabine. Br J Cancer (2003) 89:1413-7. doi:10.1038/sj.bjc.6601263

33. Bockhorn M, Uzunoglu FG, Adham M, Imrie C, Milicevic M, Sandberg AA, et al. Borderline resectable pancreatic cancer: a consensus statement by the International Study Group of Pancreatic Surgery (ISGPS). Surgery (2014) 155:977-88. doi:10.1016/j.surg.2014.02.001

34. He W, Zhao H, Chan W, Lopez D, Shroff RT, Giordano SH. Underuse of surgical resection among elderly patients with early-stage pancreatic cancer. Surgery (2015) 158:1226-34. doi:10.1016/j.surg.2015.04.031

Conflict of Interest Statement: The authors declare that the research was conducted in the absence of any commercial or financial relationships that could be construed as a potential conflict of interest.

Copyright (C) 2017 Sutera, Bernard, Gill, Harper, Quan, Bahary, Burton, Zeh and Heron. This is an open-access article distributed under the terms of the Creative Commons Attribution License (CC BY). The use, distribution or reproduction in other forums is permitted, provided the original author(s) or licensor are credited and that the original publication in this journal is cited, in accordance with accepted academic practice. No use, distribution or reproduction is permitted which does not comply with these terms. 